Homology, Homotopy and Applications, vol.15(2), 2013, pp.245-265

\title{
THE SYMMETRIC JOIN OPERAD
}

\author{
MICHAE ADAMASZEK AND JOHN D.S. JONES
}

(communicated by Jean-Louis Loday and Donald M. Davis)

\begin{abstract}
The join operad arises from the combinatorial study of the iterated join of simplices. We study a suitable simplicial version of this operad that includes the symmetries given by permutations of the factors of the join. This is the symmetric join operad. As an application of this combinatorics we construct an $E_{\infty}$-operad that coacts naturally on the chains of a simplicial set.
\end{abstract}

\section{Introduction}

Right from the outset we establish the following convention: we index simplices by the number of vertices, rather than by dimension; that is, we write $\Delta_{k}$ for a simplex with $k$ vertices and therefore dimension $k-1$.

Let $X$ and $Y$ be topological spaces. Then $X * Y$, the join of $X$ and $Y$, is a quotient space of $I \times X \times Y$ and so there is a canonical map

$$
I \times X \times Y \rightarrow X * Y .
$$

More generally, there are canonical maps

$$
\Delta_{k} \times X_{1} \times \cdots \times X_{k} \rightarrow X_{1} * \cdots * X_{k} .
$$

The join of two simplices is a simplex and these canonical maps make the sequence of spaces $\Delta_{k}$ into a topological operad.

If we look for a version of these canonical maps at the level of simplicial sets we are led, quite naturally, to two fundamental points.

- The simplest way to construct such maps for simplicial sets uses exactly the same geometric constructions with simplices that are the basis of Steenrod's construction [19] of the cup-i products at the cochain level.

- While the join operation of spaces is symmetric, it is not symmetric at the level of simplicial sets. The basic point is that if $A$ and $B$ are geometric simplices and if we order the vertices of $A$ and $B$, then the natural orderings of the vertices of $A * B$ and $B * A$ are not the same.

In the theory of classical simplicial complexes, simplices are uniquely determined by their vertices and so as usual we identify a simplex with its set of vertices. Then,

Received November 9, 2011, revised March 19, 2013; published on November 28, 2013. 2010 Mathematics Subject Classification: 18D50, 55U10, 55U15

Key words and phrases: join, operads, $E_{\infty}$, cochain operations

Article available at http://intlpress.com/HHA/v15/n2/a15 and doi:10.4310/HHA.2013.v15.n2.a15

Copyright (C) 2013, International Press. Permission to copy for private use granted. 
assuming $K$ and $L$ are classical simplicial complexes, the simplices of $K * L$ are given by $\sigma \sqcup \tau$, where $\sigma$ is a simplex in $K$ or else it is empty and $\tau$ is a simplex in $L$ or else it is empty. The simplest way of dealing with this is to add to both $K$ and $L$ a simplex of dimension -1 with the empty set as its set of vertices. Passing to simplicial sets, this amounts to working with simplicial sets augmented by adjoining a set of simplices that have "no vertices" or dimension -1 . In terms of the category $\Delta$ of ordered sets and order preserving maps used in the theory of simplicial sets, this corresponds to replacing $\Delta$ by the category $\mathcal{O}$ obtained by adjoining to $\Delta$ an additional initial object and working with $\mathcal{O}$-sets, also known as augmented simplicial sets, rather than $\Delta$-sets. The category of $\mathcal{O}$-sets is the natural context for the join operation; see $\S 3$ for the details.

However the join of $\mathcal{O}$-sets is not symmetric. What we lack is an action of the symmetric group $\Sigma_{n}$ on the set of simplices with $n$ vertices which "behaves like" reordering the vertices of an ordered simplex. This brings us to the framework of $\mathcal{O} \Sigma$-sets, an augmented version of $\Delta \Sigma$-sets, one of the basic examples of crossed simplicial groups studied by Fiedorowicz and Loday [11]. Any augmented simplicial set determines a free $\mathcal{O} \Sigma$-set, and the category of $\mathcal{O}$-sets is equivalent to the category of $\mathcal{O} \Sigma$-sets in a suitable homotopy theoretic sense; see 2.4. This leads to the symmetric join $J^{\Sigma}$ of $\mathcal{O} \Sigma$-sets and symmetric versions of the canonical maps

$$
\mathcal{O} \Sigma_{n} \times X_{1} \times \cdots \times X_{n} \rightarrow J^{\Sigma}\left(X_{1}, \ldots, X_{n}\right)
$$

where $\mathcal{O} \Sigma_{n}$ is the $\mathcal{O} \Sigma$-set corresponding to the augmented simplex $\Delta_{n}$. The join of $\mathcal{O}$-sets or $\mathcal{O} \Sigma$-sets is in fact nothing but a Day convolution on the respective presheaf category.

The join of $\mathcal{O} \Sigma_{n}$ and $\mathcal{O} \Sigma_{m}$ is $\mathcal{O} \Sigma_{n+m}$ and these constructions produce an operad $\left\{\mathcal{O} \Sigma_{n}\right\}_{n \geqslant 0}$ in the category of $\mathcal{O} \Sigma$-sets. This is the fundamental object in this paper. The other operads we consider are derived from this one by forming pairs, applying the forgetful functor to simplicial sets, and applying chains. Combining the canonical maps with the diagonal $X \rightarrow X^{n}$ we get a canonical "coaction"

$$
\mathcal{O} \Sigma_{n} \times X \rightarrow J^{\Sigma}(X, \ldots, X)
$$

on any $\mathcal{O} \Sigma$-set $X$.

Having sorted out the combinatorics of the symmetric join operad of $\mathcal{O} \Sigma$-sets, we convert it into a chain level $E_{\infty}$-operad $\{\mathfrak{j}(n)\}_{n \geqslant 0}$; see Definition 4.4. The canonical maps yield, after some manipulation, chain level coaction

$$
\mathfrak{j}(n) \otimes C_{*}(Y) \rightarrow C_{*}(Y)^{\otimes n}
$$

for any simplicial set $Y$, making $C_{*}(Y)$ into a coalgebra over $\mathfrak{j}$.

The cooperations in $C_{*}(Y)$ induced by this coaction include the classical AlexanderWhitney coproduct and higher cup-i coproducts whose duals in $C^{*}(Y)$ are the standard cup product and higher cup-i products. This expresses in a very precise sense the relation between Steenrod's construction of the cup-i products and the combinatorics of the join of simplices that was the starting point for this work.

This is not at all the first construction of a chain $E_{\infty}$-operad that endows the chain complex $C_{*}(Y)$ (resp. $C^{*}(Y)$ ) with a natural structure of an $E_{\infty}$-coalgebra (resp. $E_{\infty^{-}}$ algebra). In [17] McClure and Smith constructed the sequence operad and its action on $C^{*}(Y)$. Berger and Fresse [5] analyse the Barratt-Eccles operad, which is the 
chain version of a simplicial operad obtained from the bar constructions of symmetric groups. Another such operad is the condensation of the multi-coloured lattice path operad of Batanin and Berger [1]. It contains the sequence operad as a suboperad. There is also a significant amount of unpublished work due, independently, to Jim Milgram and Ezra Getzler to be acknowledged. These constructions are significant since by $[\mathbf{1 3}, \mathbf{1 4}]$, under suitable assumptions on $Y$, an $E_{\infty}$-algebra structure on $C^{*}(Y)$ determines the homotopy type of $Y$.

Perhaps the main theme in this paper is to understand better the relation between chain cooperations (or cochain operations) and the combinatorics of joins. Another important theme is the idea that $\mathcal{O} \Sigma$-sets give a conceptual way of encoding the relations between reorderings of the vertices of a simplex and its faces and degeneracies and therefore form the most natural setting for our general constructions. Finally, one of our aims is to do as much as possible in the context of simplicial geometry and translate this into a coaction on chains at the last possible moment.

This paper is set out as follows: $\S 2$ contains the background on the necessary modifications of simplicial sets; that is, $\mathcal{O}$-sets and $\mathcal{O} \Sigma$-sets that are the natural setting for our constructions; $\S 3$ describes in detail the construction of the symmetric join operad; in $\S 4$ we describe the manipulations required to produce and $E_{\infty}$-operad and its coaction on $C_{*}(Y)$, where $Y$ is a simplicial set; finally $\S 5$ contains some deferred proofs.

\section{Background}

\subsection{Indexing categories.}

Let $\Delta$ denote the usual simplicial category, with objects $[n]=\{0, \ldots, n\}$ for $n \geqslant 0$ and order-preserving maps as morphisms. For $0 \leqslant i \leqslant n$ let $\delta_{i}:[n-1] \rightarrow[n]$ be the injection whose image does not contain $i$ and let $\sigma_{i}:[n+1] \rightarrow[n]$ be the surjection which maps $i$ and $i+1$ to the same value.

Let $\mathcal{O}$ be the ordinal category whose objects are the sets $\bar{n}=\{1, \ldots, n\}$ for $n \geqslant 0$, where $\overline{0}=\emptyset$, and whose morphisms are the order-preserving maps. For $1 \leqslant i \leqslant n$ let $\delta_{i}: \overline{n-1} \rightarrow \bar{n}$ be the injection whose image does not contain $i$ and let $\sigma_{i}: \overline{n+1} \rightarrow \bar{n}$ be the surjection that maps $i$ and $i+1$ to the same value. For each $n$ there is a unique morphism $\overline{0} \rightarrow \bar{n}$ and there are no morphisms $\bar{n} \rightarrow \overline{0}$ if $n>0$.

The assignment $[n] \rightarrow \overline{n+1}$ defines an inclusion $\Delta \hookrightarrow \mathcal{O}$. It sends the morphisms $\delta_{i}, \sigma_{i}$ of $\Delta$ to $\delta_{i+1}, \sigma_{i+1}$ of $\mathcal{O}$, respectively.

Our main tool is the category $\mathcal{O} \Sigma$; see $[\mathbf{1 1}, \mathbf{1 8}]$. Its objects are the same as the objects of $\mathcal{O}$. A morphism $f \in \mathcal{O} \Sigma(\bar{n}, \bar{m})$ is, by definition, a map of sets together with a complete order on each of the sets $f^{-1}(i)$ for $i \in \bar{m}$. There is a forgetful functor $\mathcal{O} \Sigma \rightarrow$ Set which discards the ordering and remembers the underlying set map.

It follows that an injective set map $f: \bar{n} \rightarrow \bar{m}$ determines a unique morphism in $\mathcal{O} \Sigma$. In particular we have inclusions $\Sigma_{n} \subseteq \mathcal{O} \Sigma(\bar{n}, \bar{n})$ for all $n$. Moreover, there is a canonical embedding of categories

$$
\mathcal{O} \hookrightarrow \mathcal{O} \Sigma
$$

On the objects it is the identity and it takes an order-preserving map to the morphism with the same underlying set map and with each fibre ordered according to the natural ordering of the source. 
The category $\mathcal{O} \Sigma$ has another description. First note that any morphism $\phi \in$ $\mathcal{O} \Sigma(\bar{n}, \bar{m})$ has a unique decomposition as $\phi=f \circ \pi$, where $f \in \mathcal{O}(\bar{n}, \bar{m}) \subseteq \mathcal{O} \Sigma(\bar{n}, \bar{m})$ and $\pi \in \Sigma_{n} \subseteq \mathcal{O} \Sigma(\bar{n}, \bar{n})$. We can therefore identify elements of $\mathcal{O} \Sigma(\bar{n}, \bar{m})$ with pairs $(f, \pi) \in \mathcal{O}(\bar{n}, \bar{m}) \times \Sigma_{n}$. Let us express the composition of morphisms in this representation. First, for any permutation $\pi \in \Sigma_{n}$ and an order-preserving map $g \in \mathcal{O}(\bar{k}, \bar{n})$, define a permutation $g^{*} \pi \in \Sigma_{k}$ and a map $\pi_{*} g \in \mathcal{O}(\bar{k}, \bar{n})$ as the unique pair with the following two properties:

- the diagram

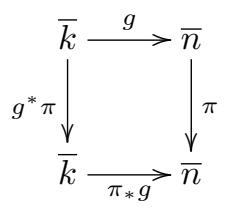

commutes

- the permutation $g^{*} \pi$ is order-preserving on each of the fibres $g^{-1}(i)$.

A quick calculation now shows that the composition of morphisms in $\mathcal{O} \Sigma$ is defined as follows: if $(f, \pi) \in \mathcal{O} \Sigma(\bar{n}, \bar{m})$ and $(g, \sigma) \in \mathcal{O} \Sigma(\bar{k}, \bar{n})$ then

$$
(f, \pi) \circ(g, \sigma)=\left(f \circ\left(\pi_{*} g\right),\left(g^{*} \pi\right) \circ \sigma\right) \in \mathcal{O} \Sigma(\bar{k}, \bar{m}) .
$$

In this representation the inclusion $\mathcal{O}(\bar{n}, \bar{m}) \hookrightarrow \mathcal{O} \Sigma(\bar{n}, \bar{m})$ is given by $f \rightarrow(f$, id $)$, the forgetful functor $\mathcal{O} \Sigma \rightarrow$ Set is given by $(f, \pi) \rightarrow f \pi$, and the inclusion $\Sigma_{n} \subseteq \mathcal{O} \Sigma(\bar{n}, \bar{n})$ is $\pi \rightarrow(\mathrm{id}, \pi)$.

\subsection{Some notation.}

Let $\mathcal{P}$ denote any of the categories $\mathcal{O}, \mathcal{O} \Sigma$, or Set. For any subset $I \subseteq \bar{m}$ let $i_{I}: \overline{|I|} \rightarrow \bar{m}$ be the unique morphism in $\mathcal{P}$ determined by the order-preserving injection of the set $\overline{|I|}$ into $\bar{m}$ with image $I$. For any morphism $f \in \mathcal{P}(\bar{k}, \bar{m})$ and any subset $I \subseteq \bar{m}$ we define $f_{I}: \overline{\left|f^{-1}(I)\right|} \rightarrow \bar{m}$ and $f^{I}: \overline{\left|f^{-1}(I)\right|} \rightarrow \overline{|I|}$ as the unique morphisms in $\mathcal{P}$ which make the following diagram commute:

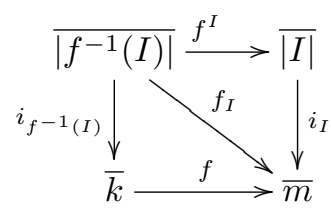

Intuitively, $f_{I}$ is the morphism $f$ restricted to the preimage of $I$ and $f^{I}$ is obtained by further restricting the target to $I$. Note that $i_{I}=\mathrm{id}_{I}$.

Now every morphism $f \in \mathcal{P}(\bar{k}, \bar{n})$ determines a decomposition of $\bar{k}$ into $n$ blocks 
$\left\{f^{-1}(i)\right\}_{i=1}^{n}$ of sizes $a_{i}=\left|f^{-1}(i)\right|$. Given a sequence of morphisms defined on these blocks,

$$
g_{i} \in \mathcal{P}\left(\overline{a_{i}}, \overline{k_{i}}\right)
$$

for some $k_{i} \geqslant 0$, we can combine them to from a morphism

$$
h \in \mathcal{P}\left(\bar{k}, \overline{k_{1}+\cdots+k_{n}}\right) .
$$

Here $h$ is the unique morphism which for $1 \leqslant i \leqslant n$ makes each of the following diagrams commute:

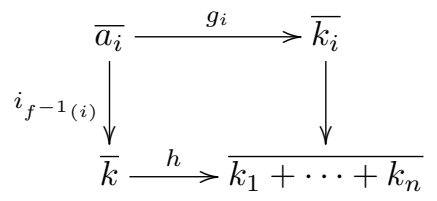

where the right hand vertical map is the order-preserving inclusion of the $i$-th block in the sum. We will use the notation

$$
h=f\left\langle g_{1}, \ldots, g_{n}\right\rangle .
$$

Note that the morphism $f$ is only used to determine the blocks, so the definition of $f\left\langle g_{1}, \ldots, g_{n}\right\rangle$ makes sense also when $f \in \operatorname{Set}(\bar{k}, \bar{n})$ and $g_{i} \in \mathcal{O} \Sigma\left(\overline{a_{i}}, \overline{k_{i}}\right)$ and it produces an element of $\mathcal{O} \Sigma\left(\bar{k}, \overline{k_{1}+\cdots+k_{n}}\right)$.

It is not difficult to verify directly that we have the following identities of $\mathcal{P}$ morphisms:

$$
\begin{aligned}
i_{I} \circ f^{I} & =f \circ i_{f-1}(I) \\
(f g)\left\langle h_{1} \circ g^{f^{-1}(1)}, \ldots, h_{n} \circ g^{f^{-1}(n)}\right\rangle & =f\left\langle h_{1}, \ldots, h_{n}\right\rangle \circ g \\
g^{f^{-1}(I)} \circ f^{I} & =(g f)^{I} \\
i_{A} \circ\left(i_{B}\right)^{A} & =i_{A \cap B} .
\end{aligned}
$$

Note that all the above constructions and the formulae $(\mathcal{P} 1)-(\mathcal{P} 4)$ are preserved by the functors $\mathcal{O} \hookrightarrow \mathcal{O} \Sigma \rightarrow$ Set.

\section{3. $\quad \Delta$-sets, $\mathcal{O}$-sets and $\mathcal{O} \Sigma$-sets.}

A $\mathcal{P}$-set (where $\mathcal{P}$ is $\Delta, \mathcal{O}$, or $\mathcal{O} \Sigma$ ) is a contravariant functor from $\mathcal{P}$ to the category of sets. We will write $X(n)$ instead of $X([n])$ or $X(\bar{n})$. It is important to be clear that for a $\Delta$-set $X$ the set $X(n)$ is to be thought of as a set of simplices of dimension $n$, while for a $\mathcal{O}$-set or $\mathcal{O} \Sigma$-set $X$ the set $X(n)$ is to be thought of as a set of simplices with $n$ vertices. For an $\mathcal{O}$-set or $\mathcal{O} \Sigma$-set $X$ we have the $i$-th face map $d_{i}=\delta_{i}^{*}: X(n) \rightarrow X(n-1)$ and $i$-th degeneracy $s_{i}=\sigma_{i}^{*}: X(n) \rightarrow X(n+1)$ for $1 \leqslant i \leqslant n$.

The inclusion $\Delta \hookrightarrow \mathcal{O}$ induces a forgetful functor $U: \mathrm{Set}^{\mathcal{O}^{\text {op }}} \rightarrow \mathrm{Set}^{\Delta^{\mathrm{op}}}$ that satisfies $(U X)(n)=X(n+1)$ for $n \geqslant 0$ (it forgets the augmentation $X(0)$ ). For a simplicial set $Y$ we will denote by $Y_{+}$the one-point augmentation; i.e., the $\mathcal{O}$-set with

$$
Y_{+}(n)= \begin{cases}Y(n-1) & \text { if } n \geqslant 1 \\ * & \text { if } n=0\end{cases}
$$

where $*$ is a singleton set. The functor $Y \mapsto Y_{+}$is the right adjoint of $U$. 
The inclusion $\mathcal{O} \hookrightarrow \mathcal{O} \Sigma$ induces another forgetful functor $I$ : Set ${ }^{\mathcal{O} \Sigma^{\text {op }}} \rightarrow \operatorname{Set}^{\mathcal{O}^{\text {op }}}$. It has a left adjoint denoted $X \mapsto X \Sigma$. For an $\mathcal{O}$-set $X$, it is given by $(X \Sigma)(n)=$ $X(n) \times \Sigma_{n}$ and the structure maps are defined by the formula

$$
(x, \pi) \circ(g, \sigma)=\left(x \circ\left(\pi_{*} g\right),\left(g^{*} \pi\right) \circ \sigma\right)
$$

for $(x, \pi) \in X(n) \times \Sigma_{n}$ and $(g, \sigma) \in \mathcal{O} \Sigma(\bar{m}, \bar{n})$. We write the structure maps as acting on the right to indicate contravariance.

In particular, for $1 \leqslant i \leqslant n$ the face map $d_{i}:(X \Sigma)(n) \rightarrow(X \Sigma)(n-1)$ is given by

$$
d_{i}(x, \pi)=(x, \pi) \circ\left(\delta_{i}, \mathrm{id}_{n-1}\right)=\left(x \circ \pi_{*} \delta_{i}, \delta_{i}^{*} \pi\right)=\left(x \delta_{\pi(i)}, \delta_{i}^{*} \pi\right)=\left(d_{\pi(i)} x, d_{i} \pi\right),
$$

where $d_{i} \pi$ denotes the $(n-1)$-permutation obtained from $\pi$ by erasing the $i$-th position and reindexing.

For each $n \geqslant 0$ we have the canonical objects $\Delta_{n}, \mathcal{O}_{n}$, and $\mathcal{O} \Sigma_{n}$ given by

$$
\Delta_{n}(m)=\Delta([m],[n]), \quad \mathcal{O}_{n}(m)=\mathcal{O}(\bar{m}, \bar{n}), \quad \mathcal{O} \Sigma_{n}(m)=\mathcal{O} \Sigma(\bar{m}, \bar{n})
$$

and

$$
\left(\Delta_{n}\right)_{+}=\mathcal{O}_{n+1}, \quad U \mathcal{O}_{n}=\Delta_{n-1} \text { for } n \geqslant 1, \quad \mathcal{O}_{n} \Sigma=\mathcal{O} \Sigma_{n} .
$$

We can also consider categories of pairs $\left(X, X^{\prime}\right)$, where $X^{\prime}$ is a sub-object of $X$. Note that since there are no morphisms in $\mathcal{O}$ or $\mathcal{O} \Sigma$ with target $\overline{0}$, the 0 -component $X(0)$ of an $\mathcal{O}$-set or $\mathcal{O} \Sigma$-set $X$ is a sub-object in a trivial way, so we can always form a pair $(X, X(0))$ of $\mathcal{O}$-sets or $\mathcal{O} \Sigma$-sets. This gives a way to remove the simplices with no vertices from either $\mathcal{O}$-sets or $\mathcal{O} \Sigma$-sets.

For every $n \geqslant 0$ we have canonical pairs

$$
\left(\Delta_{n}, \partial \Delta_{n}\right), \quad\left(\mathcal{O}_{n}, \partial \mathcal{O}_{n}\right), \quad\left(\mathcal{O} \Sigma_{n}, \partial \mathcal{O} \Sigma_{n}\right)
$$

in the respective categories. In each case the sub-object $\partial \mathcal{P}_{n}$ consists of those morphisms whose underlying set map is not surjective.

The geometric realization $|X|$ of an $\mathcal{O}$-set or $\mathcal{O} \Sigma$-set $X$ is defined by passing to the underlying simplicial set, resp., $U X$ or $U I X$.

\subsection{Homotopical properties of $\mathcal{O} \Sigma$-sets.}

Let $X$ be a $\mathcal{O}$-set and let $\eta_{X}: X \rightarrow I(X \Sigma)$ be the unit of the adjunction $(-) \Sigma:$ Set $^{\mathcal{O}^{\text {op }}} \rightleftarrows \operatorname{Set}^{\mathcal{O} \Sigma^{\text {op }}}: I$. By [11, Prop.5.1] there is a commutative diagram

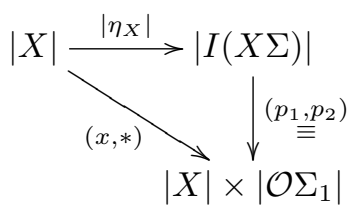

where $\left(p_{1}, p_{2}\right)$ is a homeomorphism and $\left|\mathcal{O} \Sigma_{1}\right|$ is contractible by the argument of [11, Ex.6]. It follows that $\left|\eta_{X}\right|:|X| \rightarrow|I(X \Sigma)|$ is always a homotopy equivalence. In particular, the spaces $\left|\mathcal{O} \Sigma_{n}\right|$ are contractible for $n \geqslant 1$.

In fact, one can say more. The category of $\Delta \Sigma$-sets, the obvious non-augmented version of $\mathcal{O} \Sigma$-sets, satisfies the assumptions of Theorem 6.2 of $[\mathbf{8}]$, which provides it with a model structure in which a map is a weak equivalence if and only if it is a weak equivalence of the underlying simplicial sets. Then the adjoint pair $(-) \Sigma$ : $\operatorname{Set}^{\Delta^{\mathrm{op}}} \rightleftarrows$ 
$\operatorname{Set}^{\Delta \Sigma^{\text {op }}}: I$ is a Quillen equivalence of model categories, in particular it induces an equivalence of homotopy categories.

\section{The join operad of $\mathcal{O} \Sigma$-sets.}

\subsection{Joins.}

The category of $\mathcal{O}$-sets is a natural context for the join operation at the level of simplicial sets. Let $X_{1}, \ldots, X_{n}$ be $\mathcal{O}$-sets. Then their join is the $\mathcal{O}$-set $J^{\mathcal{O}}\left(X_{1}, \ldots, X_{n}\right)$ defined as

$$
\begin{aligned}
J^{\mathcal{O}}\left(X_{1}, \ldots, X_{n}\right)(k) & =\coprod_{a_{1}+\cdots+a_{n}=k} X_{1}\left(a_{1}\right) \times \cdots \times X_{n}\left(a_{n}\right) \\
& =\coprod_{\phi \in \mathcal{O}(\bar{k}, \bar{n})} \prod_{i=1}^{n} X_{i}\left(\left|\phi^{-1}(i)\right|\right) .
\end{aligned}
$$

The two definitions are clearly equivalent because every order-preserving map $\phi \in$ $\mathcal{O}(\bar{k}, \bar{n})$ determines, and is determined by, an ordered partition of $k$ into $n$ parts of sizes $a_{i}=\left|\phi^{-1}(i)\right|$. The join has the following structure maps. An order-preserving map $f \in \mathcal{O}\left(\overline{k^{\prime}}, \bar{k}\right)$ and a partition $a_{1}+\cdots+a_{n}=k$ of $k$ determine a new partition $a_{1}^{\prime}+\cdots+a_{n}^{\prime}=k^{\prime}$ of $k^{\prime}$ and a sequence of order-preserving maps $f_{i}: \overline{a_{i}^{\prime}} \rightarrow \overline{a_{i}}$. Then for $\left(x_{1}, \ldots, x_{n}\right) \in X_{1}\left(a_{1}\right) \times \cdots \times X_{n}\left(a_{n}\right)$, we have

$$
\left(x_{1}, \ldots, x_{n}\right) \circ f=\left(x_{1} f_{1}, \ldots, x_{n} f_{n}\right) \in X\left(a_{1}^{\prime}\right) \times \cdots \times X\left(a_{n}^{\prime}\right) .
$$

There is an alternative formulation of this recipe which uses the definition (5) of the join. If $f \in \mathcal{O}\left(\overline{k^{\prime}}, \bar{k}\right)$ then $f$ takes the summand indexed by $\phi \in \mathcal{O}(\bar{k}, \bar{n})$ to the summand of $\phi f \in \mathcal{O}\left(\overline{k^{\prime}}, \bar{n}\right)$ as follows:

$$
\left(x_{1}, \ldots, x_{n}\right) \circ f=\left(x_{1} f^{\phi^{-1}(1)}, \ldots, x_{n} f^{\phi^{-1}(n)}\right) \in \prod_{i=1}^{n} X_{i}\left(\left|(\phi f)^{-1}(i)\right|\right) .
$$

Note that $f^{\phi^{-1}(i)}$ is exactly the morphism $f_{i}$ from the previous definition. It is straightforward to check using $(\mathcal{P} 3)$ that these maps define the structure of an $\mathcal{O}$-set on the join.

Notice how this definition mimics the combinatorial structure of the join of two classical simplicial complexes. Indeed it follows from [10] that if $Y_{1}, \ldots, Y_{n}$ are simplicial sets we have a homeomorphism

$$
\left|J^{\mathcal{O}}\left(Y_{1+}, \ldots, Y_{n+}\right)\right| \equiv\left|Y_{1}\right| * \cdots *\left|Y_{n}\right| .
$$

The category of $\mathcal{O} \Sigma$-sets also has a join operation. For $\mathcal{O} \Sigma$-sets $X_{1}, \ldots, X_{n}$ we define

$$
\begin{aligned}
J^{\Sigma}\left(X_{1}, \ldots, X_{n}\right)(k) & =\coprod_{a_{1}+\cdots+a_{n}=k} X_{1}\left(a_{1}\right) \times \cdots \times X_{n}\left(a_{n}\right) \times \Sigma_{a_{1}} \times \cdots \times \Sigma_{a_{n}} \Sigma_{k} \\
& =\coprod_{\phi \in \operatorname{Set}(\bar{k}, \bar{n})} \prod_{i=1}^{n} X_{i}\left(\left|\phi^{-1}(i)\right|\right) .
\end{aligned}
$$

The equivalence of the two definitions follows from the fact that every map of sets $\phi \in \operatorname{Set}(\bar{k}, \bar{n})$ can be factored as $\phi=f \pi$ with $\pi \in \Sigma_{k}$ and $f \in \mathcal{O}(\bar{k}, \bar{n})$. In this 
factorization, $f$ is determined uniquely and it induces a decomposition $a_{1}+\cdots+a_{n}=$ $k$ (with $a_{i}=\left|\phi^{-1}(i)\right|=\left|f^{-1}(i)\right|$ ), while $\pi$ is unique up to postcomposition with an element of $\Sigma_{a_{1}} \times \cdots \times \Sigma_{a_{n}}$. The maps

$$
\begin{gathered}
\left(x_{1}, \ldots, x_{n}\right) \rightarrow\left[\left(x_{1}\left(\pi^{-1}\right)^{\phi^{-1}(1)}, \ldots, x_{n}\left(\pi^{-1}\right)^{\phi^{-1}(n)}\right), \pi\right] \\
{\left[\left(y_{1}, \ldots, y_{n}\right), \pi\right] \rightarrow\left(y_{1} \pi^{f^{-1}(1)}, \ldots, y_{n} \pi^{f^{-1}(n)}\right)}
\end{gathered}
$$

establish the equivalence between the two versions of the join.

The structure map of $J^{\Sigma}$ induced by the morphism $f \in \mathcal{O} \Sigma\left(\overline{k^{\prime}}, \bar{k}\right)$ takes the summand indexed by $\phi \in \operatorname{Set}(\bar{k}, \bar{n})$ to the summand of $\phi f \in \operatorname{Set}\left(\overline{k^{\prime}}, \bar{n}\right)$ by the formula

$$
\left(x_{1}, \ldots, x_{n}\right) \circ f=\left(x_{1} f^{\phi^{-1}(1)}, \ldots, x_{n} f^{\phi^{-1}(n)}\right) \in \prod_{i=1}^{n} X_{i}\left(\left|(\phi f)^{-1}(i)\right|\right) .
$$

The verification that these maps assemble to the structure of an $\mathcal{O} \Sigma$-set is identical to that for $J^{\mathcal{O}}$.

A direct calculation with the representation (6) of the join shows that if $f=$ $(g, \sigma) \in \mathcal{O}\left(\overline{k^{\prime}}, \bar{k}\right) \times \Sigma_{k^{\prime}}$ then the structure map induced by $f$ is given by the formula

$$
\left[\left(x_{1}, \ldots, x_{n}\right), \pi\right] \circ(g, \sigma)=\left[\left(x_{1}\left(\pi_{*} g\right)_{1}, \ldots, x_{n}\left(\pi_{*} g\right)_{n}\right), g^{*} \pi \circ \sigma\right]
$$

where $\left(\pi_{*} g\right)_{i}: \overline{a_{i}^{\prime}} \rightarrow \overline{a_{i}}$ are the components of the order-preserving map $\pi_{*} g: \overline{k^{\prime}} \rightarrow \bar{k}$ determined by the partition $a_{1}+\cdots+a_{n}=k$.

Lemma 3.1. For any sequence of $\mathcal{O}$-sets $X_{1}, \ldots, X_{n}$, we have an isomorphism of $\mathcal{O} \Sigma$-sets

$$
J^{\Sigma}\left(X_{1} \Sigma, \ldots, X_{n} \Sigma\right)=J^{\mathcal{O}}\left(X_{1}, \ldots, X_{n}\right) \Sigma .
$$

Proof. Since $X_{i} \Sigma(k)=X_{i}(k) \times \Sigma_{k}$ this follows immediately from (6) and (4).

Because every map $h \in \mathcal{O}\left(\bar{k}, \overline{k_{1}+\cdots+k_{n}}\right)$ has a unique presentation in the form $h=f\left\langle g_{1}, \ldots, g_{n}\right\rangle$, where $f \in \mathcal{O}(\bar{k}, \bar{n})$ and $g_{i} \in \mathcal{O}\left(\overline{\left|f^{-1}(i)\right|}, \overline{k_{i}}\right)$, we obtain isomorphisms

$$
\Theta: J^{\mathcal{O}}\left(\mathcal{O}_{k_{1}}, \ldots, \mathcal{O}_{k_{n}}\right) \stackrel{\simeq}{\longrightarrow} \mathcal{O}_{k_{1}+\cdots+k_{n}} .
$$

It follows from Lemma 3.1 that they yield isomorphisms

$$
\Theta: J^{\Sigma}\left(\mathcal{O} \Sigma_{k_{1}}, \ldots, \mathcal{O} \Sigma_{k_{n}}\right) \stackrel{\simeq}{\longrightarrow} \mathcal{O} \Sigma_{k_{1}+\cdots+k_{n}}
$$

In both cases the map inducing the isomorphism acts on the summand of the join indexed by a map $\phi \in \operatorname{Set}(\bar{k}, \bar{n})$ as

$$
\prod_{i=1}^{n} \mathcal{P}\left(\overline{\left|\phi^{-1}(i)\right|}, \overline{k_{i}}\right) \ni\left(g_{1}, \ldots, g_{n}\right) \stackrel{\Theta}{\rightarrow} \phi\left\langle g_{1}, \ldots, g_{n}\right\rangle \in \mathcal{P}\left(\bar{k}, \overline{k_{1}+\cdots+k_{n}}\right),
$$

where $\mathcal{P}$ is $\mathcal{O}$ or $\mathcal{O} \Sigma$. We will frequently use the formal similarity between (5) and (7) to present a single argument that simultaneously applies to analogous statements about $J^{\mathcal{O}}$ and $J^{\Sigma}$.

\subsection{Day convolution, monoidal structure and symmetry.}

The join operations in $\mathcal{O}$-sets and $\mathcal{O} \Sigma$-sets are instances of a standard construction known as Day convolution $[\mathbf{6}, \mathbf{2}, \mathbf{7}]$, a monoidal product in a presheaf category. More 
precisely, if $(\mathcal{C}, \otimes, I)$ is a small monoidal category then the category of functors Set $^{\mathcal{C}}$ becomes a closed monoidal category with the convolution product given by the coend

$$
\left(X_{1} \otimes \cdots \otimes X_{n}\right)(c)=\int^{a_{1}, \ldots, a_{n}} X_{1}\left(a_{1}\right) \times \cdots \times X_{n}\left(a_{n}\right) \times \mathcal{C}\left(a_{1} \otimes \cdots \otimes a_{n}, c\right)
$$

for $X_{1}, \ldots, X_{n} \in \operatorname{Set}^{\mathcal{C}}$. Moreover, if $\mathcal{C}$ is symmetric, then so is $\operatorname{Set}^{\mathcal{C}}$. The categories $\mathcal{C}=$ $\mathcal{O}^{\text {op }}$ and $\mathcal{C}=\mathcal{O} \Sigma^{\mathrm{op}}$ are monoidal categories under the ordered union $m \otimes n=m \sqcup n$, and one checks directly that the convolution products in $\operatorname{Set}^{\mathcal{O}^{\text {op }}}$ and $\operatorname{Set}^{\mathcal{O} \Sigma^{\text {op }}}$ amount to the joins $J^{\mathcal{O}}$ and $J^{\Sigma}$ of (4) and (6). Their units are $\mathcal{O}_{0}$ and $\mathcal{O} \Sigma_{0}$, respectively.

It follows from the general properties of Day convolution that the join operation is associative. We state this fact explicitly just to introduce the notation that will be used later.

Lemma 3.2. Let $X_{1, *}, \ldots, X_{n, *}$ be sequences of $\mathcal{O}$-sets or $\mathcal{O} \Sigma$-sets, where the $i$-th sequence has length $k_{i}$ for $i=1, \ldots, n$. Let $X_{*, *}$ denote the sequence of length $\sum k_{i}$ obtained by joining the given sequences in the lexicographic order of indices. Then there is an isomorphism

$$
\Theta: J\left(J\left(X_{1, *}\right), \ldots, J\left(X_{n, *}\right)\right) \rightarrow J\left(X_{*, *}\right),
$$

where $J=J^{\mathcal{O}}$ or $J=J^{\Sigma}$.

From now we will often simplify notation in this way by writing $X_{*}$ instead of $\left(X_{1}, \ldots, X_{n}\right)$.

The join of $\mathcal{O}$-sets, however, is not symmetric, which is a consequence of the fact that $\mathcal{O}$ is not symmetric. Indeed, the $\mathcal{O}$-sets $J^{\mathcal{O}}(X, Y)$ and $J^{\mathcal{O}}(Y, X)$ have isomorphic sets of simplices but there is no isomorphism between these sets that commutes with the structure maps. Intuitively, they have the same simplices but the vertices of these simplices are ordered differently. Since the category $\mathcal{O} \Sigma^{\mathrm{op}}$ is symmetric monoidal, this deficiency is corrected by the join $J^{\Sigma}$ of $\mathcal{O} \Sigma$-sets. For any permutation $\sigma \in \Sigma_{n}$, one can explicitly describe the natural isomorphism

$$
T_{\sigma}: J^{\Sigma}\left(X_{1}, \ldots, X_{n}\right) \rightarrow J^{\Sigma}\left(X_{\sigma^{-1}(1)}, \ldots, X_{\sigma^{-1}(n)}\right)
$$

of $\mathcal{O} \Sigma$-sets. Consider the presentation (7) of the join. In degree $k$ the map $T_{\sigma}$ sends the summand of $J^{\Sigma}\left(X_{*}\right)$ indexed by $\phi \in \operatorname{Set}(\bar{k}, \bar{n})$ to the summand of $J^{\Sigma}\left(X_{\sigma^{-1}(*)}\right)$ indexed by $\sigma \phi \in \operatorname{Set}(\bar{k}, \bar{n})$, shuffling the factors appropriately.

We can transcribe this description of the isomorphism $T_{\sigma}$ to the presentation (6). Given $\sigma \in \Sigma_{n}$ and $a_{1}+\cdots+a_{n}=k$, let $\sigma_{a_{1}, \ldots, a_{n}} \in \Sigma_{k}$ be the block permutation determined by $\sigma$ that permutes the blocks of sizes $a_{1}, \ldots, a_{n}$ in the way $\sigma$ permutes $n$ letters. Formally, $\sigma_{a_{1}, \ldots, a_{n}}=\sigma_{*} f$, where $f \in \mathcal{O}(\bar{k}, \bar{n})$ is the order-preserving map corresponding to the partition $a_{1}+\cdots+a_{n}=k$. Then

$$
T_{\sigma}\left(\left[\left(x_{1}, \ldots, x_{n}\right), \pi\right]\right)=\left[\left(x_{\sigma^{-1}(1)}, \ldots, x_{\sigma^{-1}(n)}\right), \sigma_{a_{1}, \ldots, a_{n}} \pi\right] .
$$

In particular, the basic symmetry operator of the monoidal structure

$$
T_{X, Y}: J^{\Sigma}(X, Y) \rightarrow J^{\Sigma}(Y, X)
$$

acts by sending the element $[(x, y), \pi] \in X(a) \times Y(b) \times_{\Sigma_{a} \times \Sigma_{b}} \Sigma_{a+b}$ to

$$
\left[(y, x), \tau_{a, b} \pi\right] \in Y(b) \times X(a) \times_{\Sigma_{b} \times \Sigma_{a}} \Sigma_{b+a},
$$

where $\tau_{a, b}$ switches the two blocks of sizes $a$ and $b$. 


\subsection{Canonical maps and the join operad of $\mathcal{O} \Sigma$-sets.}

We can now define the $\mathcal{O} \Sigma$-analogue of the canonical maps of the introduction. There are obvious maps

$$
\Psi_{k_{1}, \ldots, k_{n}}: \mathcal{O} \Sigma_{n} \times \mathcal{O} \Sigma_{k_{1}} \times \cdots \times \mathcal{O} \Sigma_{k_{n}} \rightarrow J^{\Sigma}\left(\mathcal{O} \Sigma_{k_{1}}, \ldots, \mathcal{O} \Sigma_{k_{n}}\right)=\mathcal{O} \Sigma_{k_{1}+\cdots+k_{n}}
$$

given by the formula

$$
\Psi_{k_{1}, \ldots, k_{n}}\left(f ; g_{1}, \ldots, g_{n}\right)=f\left\langle g_{1} \circ i_{f^{-1}(1)}, \ldots, g_{n} \circ i_{f^{-1}(n)}\right\rangle \in \mathcal{O} \Sigma\left(\bar{k}, \overline{k_{1}+\cdots+k_{n}}\right)
$$

for $\left(f ; g_{1}, \ldots, g_{n}\right) \in \mathcal{O} \Sigma(\bar{k}, \bar{n}) \times \mathcal{O} \Sigma\left(\bar{k}, \overline{k_{1}}\right) \times \cdots \times \mathcal{O} \Sigma\left(\bar{k}, \overline{k_{n}}\right)$. Our main observation in this section is that these maps define an operad.

Theorem 3.3. The sequence of $\mathcal{O} \Sigma$-sets $\left\{\mathcal{O} \Sigma_{n}\right\}_{n \geqslant 0}$ forms an operad (non-unital, with permutations) in the symmetric monoidal category of $\mathcal{O} \Sigma$-sets ( $\operatorname{Set}^{\mathcal{O} \Sigma^{\text {op }}}, \times, *$ ). The structure maps of the operad are the $\Psi_{k_{1}, \ldots, k_{n}}$ of (11). The right action of $\Sigma_{n}$ on $\mathcal{O} \Sigma_{n}$ is given by

$$
f \circ \pi=\pi^{-1} f
$$

for $f \in \mathcal{O} \Sigma_{n}(k)$ and $\pi \in \Sigma_{n}$.

The proof can be found in Section 5. It is a tedious but otherwise straightforward verification. The operad has no unit since there is no map $* \rightarrow \mathcal{O} \Sigma_{1}$ of $\mathcal{O} \Sigma$-sets.

In order to discuss operad coactions we introduce the following notation. For a sequence of $\mathcal{O} \Sigma$-sets $X_{1}, \ldots, X_{n}$ let $A\left(X_{1}, \ldots, X_{n}\right)$, also denoted $A\left(X_{*}\right)$, be the $\mathcal{O} \Sigma$ set

$$
A\left(X_{*}\right)=\mathcal{O} \Sigma_{n} \times X_{1} \times \cdots \times X_{n} .
$$

Since a natural transformation between colimit-preserving functors on presheaf categories is determined by its values on the representable objects, the maps (11) defines maps of $\mathcal{O} \Sigma$-sets

$$
\alpha: A\left(X_{*}\right) \rightarrow J^{\Sigma}\left(X_{*}\right)
$$

We can describe them explicitly. Write an element of $A\left(X_{*}\right)(k)=\mathcal{O} \Sigma(\bar{k}, \bar{n}) \times X_{1}(k) \times$ $\cdots \times X_{n}(k)$ as a tuple $\left(f ; x_{1}, \ldots, x_{n}\right)$. Then $\alpha\left(f ; x_{1}, \ldots, x_{n}\right)$ lies in the summand of $J^{\Sigma}\left(X_{*}\right)$ indexed by the underlying map $f \in \operatorname{Set}(\bar{k}, \bar{n})$ and

$$
\alpha\left(f ; x_{1}, \ldots, x_{n}\right)=\left(x_{1} i_{f^{-1}(1)}, \ldots, x_{n} i_{f^{-1}(n)}\right) .
$$

If $f=(g, \sigma)$ then we can also write

$$
\alpha\left((g, \sigma) ; x_{1}, \ldots, x_{n}\right)=\left[\left(x_{1}\left(\sigma^{-1} \circ i_{g^{-1}(1)}\right), \ldots, x_{n}\left(\sigma^{-1} \circ i_{g^{-1}(n)}\right)\right), \sigma\right] .
$$

One can immediately see that $\alpha$ is $\Sigma_{n}$-equivariant in the sense that

$$
T_{\pi}\left(\alpha\left(f ; x_{1}, \ldots, x_{n}\right)\right)=\alpha\left(\pi f ; x_{\pi^{-1}(1)}, \ldots, x_{\pi^{-1}(n)}\right) .
$$

Until now we have constructed an operad of $\mathcal{O} \Sigma$-sets that governs the relations between the canonical maps of the symmetric join $J^{\Sigma}$. In addition to the operad structure, the transformation $\alpha$, precomposed with the $n$-fold diagonal $X \rightarrow X \times$ $\cdots \times X$, yields for every $\mathcal{O} \Sigma$-set $X$ and every $n \geqslant 0$ maps of $\mathcal{O} \Sigma$-sets

$$
\Psi_{n}^{X}: \mathcal{O} \Sigma_{n} \times X \rightarrow J^{\Sigma}\left(X^{n}\right)
$$

where $J^{\Sigma}\left(X^{n}\right)=J^{\Sigma}(X, \ldots, X)$. 


\subsection{The induced operads of pairs.}

We now explain how the operad $\left\{\mathcal{O} \Sigma_{n}\right\}_{n} \geqslant 0$ gives rise to an operad in the category of pairs of $\mathcal{O} \Sigma$-sets and, via the forgetful functor $I$, an operad in the category of pairs of $\mathcal{O}$-sets.

First, given a sequence $X_{1}, \ldots, X_{n}$ of $\mathcal{O}$-sets or $\mathcal{O} \Sigma$-sets we define the relative join as the pair

$$
\left(J\left(X_{1}, \ldots, X_{n}\right), \partial J\left(X_{1}, \ldots, X_{n}\right)\right)
$$

for $J=J^{\mathcal{O}}$ or $J=J^{\Sigma}$, where $\partial J\left(X_{1}, \ldots, X_{n}\right)$ is the sub-object consisting of the summands in (5) or (7) indexed by non-surjective maps $\phi$. In other words, those are the simplices of the join that do not contain a proper face from at least one of the factors.

The maps $\Psi_{k_{1}, \ldots, k_{n}}$ of (11) clearly induce maps of relative $\mathcal{O} \Sigma$-sets

$$
\begin{aligned}
\Psi_{k_{1}, \ldots, k_{n}}:\left(\mathcal{O} \Sigma_{n}, \partial \mathcal{O} \Sigma_{n}\right) & \times\left(\mathcal{O} \Sigma_{k_{1}}, \partial \mathcal{O} \Sigma_{k_{1}}\right) \times \cdots \times\left(\mathcal{O} \Sigma_{k_{n}}, \partial \mathcal{O} \Sigma_{k_{n}}\right) \\
& \rightarrow\left(\mathcal{O} \Sigma_{k_{1}+\cdots+k_{n}}, \partial \mathcal{O} \Sigma_{k_{1}+\cdots+k_{n}}\right)
\end{aligned}
$$

and it is an immediate corollary of Theorem 3.3 that $\left\{\left(\mathcal{O} \Sigma_{n}, \partial \mathcal{O} \Sigma_{n}\right)\right\}_{n \geqslant 0}$ is an operad in the category of pairs. Moreover, in the relative context the map $\alpha$ induces a map

$$
\alpha:\left(\mathcal{O} \Sigma_{n}, \partial \mathcal{O} \Sigma_{n}\right) \times\left(X_{1}, X_{1}(0)\right) \times \cdots \times\left(X_{n}, X_{n}(0)\right) \rightarrow\left(J^{\Sigma}\left(X_{*}\right), \partial J^{\Sigma}\left(X_{*}\right)\right),
$$

which specializes to a relative version of (16)

$$
\Psi_{n}^{X}:\left(\mathcal{O} \Sigma_{n}, \partial \mathcal{O} \Sigma_{n}\right) \times(X, X(0)) \rightarrow\left(J^{\Sigma}\left(X^{n}\right), \partial J^{\Sigma}\left(X^{n}\right)\right) .
$$

\section{The chain operad}

In this section we explain how to construct an $E_{\infty}$-operad $\mathfrak{j}$ in the category of chain complexes from the symmetric join operad. We go on to explain how the maps $\Psi_{n}^{X}$ of (19) give a coaction of the operad $\mathfrak{j}$ on the chain complex $C_{*}(Y)$ of a simplicial set $Y$.

\subsection{Conventions regarding chain complexes.}

We fix once for all some commutative ground ring $k$. Let $(\mathbf{C h}, \otimes, k)$ denote the symmetric monoidal category of chain complexes of $k$-modules, with differential of degree -1 . The symmetry operator is

$$
T(x \otimes y)=(-1)^{\operatorname{deg}(x) \operatorname{deg}(y)} y \otimes x .
$$

For a chain complex $(C, d)$, let $\sigma^{n} C$ denote the $n$-fold chain suspension; i.e., the chain complex with $\left(\sigma^{n} C\right)_{m}=C_{m-n}$ and differential $d\left(\sigma^{n} x\right)=(-1)^{n} \sigma^{n} d x$. For any chain complexes $C$ and $D$ we have an isomorphism

$$
\sigma^{n} C \otimes \sigma^{m} D=\sigma^{n+m}(C \otimes D)
$$

given by $\sigma^{n} x \otimes \sigma^{m} y \rightarrow(-1)^{m \operatorname{deg}(x)} \sigma^{n+m}(x \otimes y)$. 
If $Y$ is a simplicial set then $C_{*}(Y)$ denotes its usual chain complex. The functor

$$
C_{*}(-):\left(\operatorname{Set}^{\Delta^{\mathrm{op}}}, \times, *\right) \rightarrow(\mathbf{C h}, \otimes, k)
$$

is lax-monoidal with the natural transformation

$$
C_{*}\left(Y_{1}\right) \otimes C_{*}\left(Y_{2}\right) \stackrel{E Z}{\longrightarrow} C_{*}\left(Y_{1} \times Y_{2}\right)
$$

given by the Eilenberg-Zilber map [16, Def.29.7].

\subsection{Chain complexes of $\mathcal{O}$-sets.}

If $X$ is an $\mathcal{O}$-set, we define the augmented chain complex $C_{*}^{\mathcal{O}}(X)$ by

$$
C_{n}^{\mathcal{O}}(X)=k[X(n)]
$$

with differential $d x=\sum_{i=1}^{n}(-1)^{i} d_{i} x$ for $x \in X(n)$. If $\left(X, X^{\prime}\right)$ is an $\mathcal{O}$-set pair then we can form the relative chain complex $C_{*}^{\mathcal{O}}\left(X, X^{\prime}\right)$ in the usual way.

Recall the adjoint functors $U$ : $\operatorname{Set}^{\mathcal{O}^{\mathrm{op}}} \rightleftarrows \operatorname{Set}^{\Delta^{\mathrm{op}}}:(-)_{+}$between $\mathcal{O}$-sets and $\Delta$-sets. The sign conventions associated to suspensions imply that

$$
\begin{aligned}
C_{*}^{\mathcal{O}}(X, X(0)) & =\sigma C_{*}(U X) & & \text { for any } \mathcal{O} \text {-set } X, \\
C_{*}^{\mathcal{O}}\left(Y_{+}, Y_{+}(0)\right) & =\sigma C_{*}(Y) & & \text { for any } \Delta \text {-set } Y .
\end{aligned}
$$

Remark. The last isomorphism holds for any functorial augmentation $Y_{+}$of simplicial sets. In fact we will see that the choice of augmentation will not affect the final outcome of the constructions of this section. This is not surprising, since we are trying to produce chain level maps for $\Delta$-sets, while $\mathcal{O}$-sets and $\mathcal{O} \Sigma$-sets serve only as an intermediate tool.

We also have

$$
\begin{aligned}
C_{*}^{\mathcal{O}}\left(J^{\mathcal{O}}\left(X_{1}, \ldots, X_{n}\right)\right) & =\bigotimes_{i=1}^{n} C_{*}^{\mathcal{O}}\left(X_{i}\right) \\
C_{*}^{\mathcal{O}}\left(J^{\mathcal{O}}\left(X_{1}, \ldots, X_{n}\right), \partial J^{\mathcal{O}}\left(X_{1}, \ldots, X_{n}\right)\right) & =\bigotimes_{i=1}^{n} C_{*}^{\mathcal{O}}\left(X_{i}, X_{i}(0)\right)
\end{aligned}
$$

for any sequence $X_{1}, \ldots, X_{n}$ of $\mathcal{O}$-sets. In other words, we have a monoidal functor $C_{*}^{\mathcal{O}}(-):\left(\operatorname{Set}^{\mathcal{O}^{\text {op }}}, J^{\mathcal{O}}, \mathcal{O}_{0}\right) \rightarrow(\mathbf{C h}, \otimes, k)$. On the other hand, using the isomorphisms (20), we see that the Eilenberg-Zilber map induces in the augmented context a transformation

$$
\sigma^{-1} C_{*}^{\mathcal{O}}\left(X_{1}, X_{1}(0)\right) \otimes \sigma^{-1} C_{*}^{\mathcal{O}}\left(X_{2}, X_{2}(0)\right) \stackrel{E Z}{\longrightarrow} \sigma^{-1} C_{*}^{\mathcal{O}}\left(X_{1} \times X_{2}, X_{1}(0) \times X_{2}(0)\right),
$$

which makes the functor $\sigma^{-1} C_{*}^{\mathcal{O}}(-,-(0)):\left(\operatorname{Set}^{\mathcal{O}^{\text {op }}}, \times, *\right) \rightarrow(\mathbf{C h}, \otimes, k)$ lax-monoidal.

\subsection{Chain complexes of $\mathcal{O} \Sigma$-sets.}

If $Z$ is an $\mathcal{O} \Sigma$-set, we will continue to write $C_{*}^{\mathcal{O}}(Z)$ for the chain complex of the underlying $\mathcal{O}$-set $I Z$. Recall from 2.4 that for any $\mathcal{O}$-set $X$ the standard inclusion $\eta_{X}: X \rightarrow I(X \Sigma)$ is a weak equivalence. There is no natural inverse map $X \Sigma \rightarrow X$, but a suitable inverse exists at the level of chain complexes (see also the miraculous map of [3, Def. 4.2]). 
Proposition 4.1. For every $\mathcal{O}$-set $X$, the assignment

$$
(x, \pi) \rightarrow \operatorname{sgn}(\pi) x
$$

induces a natural map of chain complexes

$$
s_{X}: C_{*}^{\mathcal{O}}(X \Sigma) \rightarrow C_{*}^{\mathcal{O}}(X),
$$

which gives a quasi-isomorphism $C_{*}^{\mathcal{O}}(X \Sigma, X \Sigma(0)) \stackrel{\sim}{\longrightarrow} C_{*}^{\mathcal{O}}(X, X(0))$.

Proof. Let us first verify that $s=s_{X}$ is indeed a map of chain complexes. We have

$$
d s(x, \pi)=\operatorname{sgn}(\pi) d x=\operatorname{sgn}(\pi) \sum_{i=1}^{n}(-1)^{i} d_{i} x .
$$

Using (3) and the easy formula

$$
\operatorname{sgn}\left(d_{i} \pi\right)=(-1)^{i+\pi(i)} \operatorname{sgn}(\pi)
$$

we verify that

$$
\begin{aligned}
s d(x, \pi) & =s \sum_{i=1}^{n}(-1)^{i}\left(d_{\pi(i)} x, d_{i} \pi\right) \\
& =\sum_{i=1}^{n}(-1)^{i} \operatorname{sgn}\left(d_{i} \pi\right) d_{\pi(i)} x \\
& =\operatorname{sgn}(\pi) \sum_{i=1}^{n}(-1)^{\pi(i)} d_{\pi(i)} x=\operatorname{sgn}(\pi) \sum_{i=1}^{n}(-1)^{i} d_{i} x=d s(x, \pi),
\end{aligned}
$$

so the claim is proved.

The map $s_{X}$ is clearly natural. To prove that the map of relative complexes is a quasi-isomorphism note that the identity of $C_{*}^{\mathcal{O}}(X, X(0))$ factors as

$$
C_{*}^{\mathcal{O}}(X, X(0)) \stackrel{C_{*}^{\mathcal{O}}\left(\eta_{X}\right)}{\longrightarrow} C_{*}^{\mathcal{O}}(X \Sigma, X \Sigma(0)) \stackrel{s_{X}}{\longrightarrow} C_{*}^{\mathcal{O}}(X, X(0)),
$$

and $C_{*}^{\mathcal{O}}\left(\eta_{X}\right)$ is a quasi-isomorphism by the results of Section 2.4.

Consider now the chain morphism $s_{X}$ in the special case when $X=J^{\mathcal{O}}\left(X_{1}, \ldots, X_{n}\right)$. Then by Lemma 3.1 and (21), $s_{X}$ can be identified with the map

$$
C_{*}^{\mathcal{O}}\left(J^{\Sigma}\left(X_{*} \Sigma\right)\right)=C_{*}^{\mathcal{O}}\left(J^{\mathcal{O}}\left(X_{*}\right) \Sigma\right) \stackrel{s_{X}}{\longrightarrow} C_{*}^{\mathcal{O}}\left(J^{\mathcal{O}}\left(X_{*}\right)\right)=\bigotimes_{i=1}^{n} C_{*}^{\mathcal{O}}\left(X_{i}\right) .
$$

Proposition 4.2. For any $\mathcal{O}$-sets $X_{1}, \ldots, X_{n}$ the maps

$$
s_{J^{\mathcal{O}}\left(X_{*}\right)}: C_{*}^{\mathcal{O}}\left(J^{\Sigma}\left(X_{1} \Sigma, \ldots, X_{n} \Sigma\right)\right) \rightarrow \bigotimes_{i=1}^{n} C_{*}^{\mathcal{O}}\left(X_{i}\right)
$$

are $\Sigma_{n}$-equivariant. 
Proof. Due to obvious associativity it suffices to check the claim for the join of two objects; that is, to verify the commutativity of the diagram

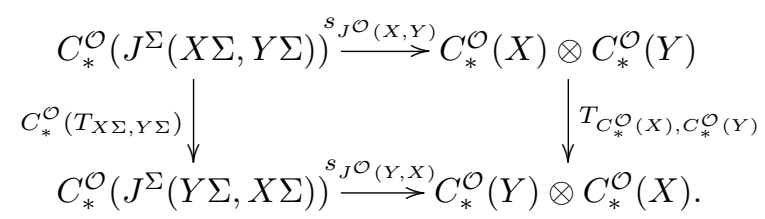

If an element of $J^{\Sigma}(X \Sigma, Y \Sigma)(k)=J^{\mathcal{O}}(X, Y) \Sigma(k)$ is represented by the triple $\left(x_{p}, y_{q}, \pi\right)$ with $p+q=k$ then

$$
T s(x, y, \pi)=\operatorname{sgn}(\pi) T(x \otimes y)=(-1)^{p q} \operatorname{sgn}(\pi) y \otimes x
$$

while

$$
s T(x, y, \pi)=s\left(y, x, \tau_{p, q} \pi\right)=\operatorname{sgn}\left(\tau_{p, q}\right) \operatorname{sgn}(\pi) y \otimes x
$$

and the two values are equal because $\operatorname{sgn}\left(\tau_{p, q}\right)=(-1)^{p q}$.

\subsection{Construction of the chain operad.}

The structure maps of the operad $\left\{\left(\mathcal{O} \Sigma_{n}, \partial \mathcal{O} \Sigma_{n}\right)\right\}_{n \geqslant 0}$ of pairs of $\mathcal{O} \Sigma$-sets (17) induce, via the lax-monoidal functor $\sigma^{-1} C_{*}^{\mathcal{O}}(-)$ of $(22)$, maps of chain complexes

$$
\begin{gathered}
\mathfrak{a}_{k_{1}, \ldots, k_{n}}: \sigma^{-1} C_{*}^{\mathcal{O}}\left(\mathcal{O} \Sigma_{n}, \partial \mathcal{O} \Sigma_{n}\right) \otimes \bigotimes_{i=1}^{n} \sigma^{-1} C_{*}^{\mathcal{O}}\left(\mathcal{O} \Sigma_{k_{i}}, \partial \mathcal{O} \Sigma_{k_{i}}\right) \\
\rightarrow \sigma^{-1} C_{*}^{\mathcal{O}}\left(\mathcal{O} \Sigma_{k_{1}+\cdots+k_{n}}, \partial \mathcal{O} \Sigma_{k_{1}+\cdots+k_{n}}\right)
\end{gathered}
$$

which are therefore the structure maps of a chain operad

$$
\mathfrak{a}(n)=\sigma^{-1} C_{*}^{\mathcal{O}}\left(\mathcal{O} \Sigma_{n}, \partial \mathcal{O} \Sigma_{n}\right), \quad n \geqslant 0 .
$$

In particular, $\mathfrak{a}(0)=\sigma^{-1} k$. It is also easy to check that if id $\in \mathcal{O} \Sigma(\overline{1}, \overline{1})$ denotes the unique morphism then $\sigma^{-1} \mathrm{id} \in \mathfrak{a}(1)_{0}$ is the unit of this operad.

Recall that for any $\mathcal{O} \Sigma$-set we have the maps of (19). Now let $X$ be an $\mathcal{O}$-set. Consider the following composition $\mathfrak{a}_{n}^{X}$.

$$
\begin{aligned}
\mathfrak{a}_{n}^{X}: \mathfrak{a}(n) \otimes C_{*}^{\mathcal{O}}(X, X(0)) \stackrel{=}{=} & \sigma^{-1} C_{*}^{\mathcal{O}}\left(\mathcal{O} \Sigma_{n}, \partial \mathcal{O} \Sigma_{n}\right) \otimes C_{*}^{\mathcal{O}}(X, X(0)) \\
\stackrel{1 \otimes \eta_{*}}{\longrightarrow} & \sigma^{-1} C_{*}^{\mathcal{O}}\left(\mathcal{O} \Sigma_{n}, \partial \mathcal{O} \Sigma_{n}\right) \otimes C_{*}^{\mathcal{O}}(X \Sigma, X \Sigma(0)) \\
\stackrel{E Z}{\longrightarrow} & C_{*}^{\mathcal{O}}\left(\left(\mathcal{O} \Sigma_{n}, \partial \mathcal{O} \Sigma_{n}\right) \times(X \Sigma, X \Sigma(0))\right) \\
\stackrel{\Psi_{n}^{X \Sigma}}{\longrightarrow} & C_{*}^{\mathcal{O}}\left(J^{\Sigma}\left(X \Sigma^{n}\right), \partial J^{\Sigma}\left(X \Sigma^{n}\right)\right) \\
= & C_{*}^{\mathcal{O}}\left(J^{\mathcal{O}}\left(X^{n}\right) \Sigma, \partial J^{\mathcal{O}}\left(X^{n}\right) \Sigma\right) \\
\stackrel{s_{J} \mathcal{O}_{\left(X^{n}\right)}}{\longrightarrow} & C_{*}^{\mathcal{O}}\left(J^{\mathcal{O}}\left(X^{n}\right), \partial J^{\mathcal{O}}\left(X^{n}\right)\right) \\
= & C_{*}^{\mathcal{O}}(X, X(0))^{\otimes n}
\end{aligned}
$$

In summary, we first enlarge $X$ to the $\mathcal{O} \Sigma$-set $X \Sigma$, apply the simplicial "coaction" maps of (19), and pass back to the non-symmetric context using Proposition 4.1. 
Proposition 4.3. For any $\mathcal{O}$-set $X$ the maps

$$
\mathfrak{a}_{n}^{X}: \mathfrak{a}(n) \otimes C_{*}^{\mathcal{O}}(X, X(0)) \rightarrow C_{*}^{\mathcal{O}}(X, X(0))^{\otimes n}
$$

equip $C_{*}^{\mathcal{O}}(X, X(0))$ with a natural structure of a coalgebra over the operad $\{\mathfrak{a}(n)\}_{n \geqslant 0}$.

For the proof see the last section.

Now suppose that $X=Y_{+}$for a simplicial set $Y$. Then $C_{*}^{\mathcal{O}}\left(Y_{+}, Y_{+}(0)\right)=\sigma C_{*}(Y)$ and we conclude that $\sigma C_{*}(Y)$ is a coalgebra over $\mathfrak{a}$. Now we use the device known as operadic desuspension of $\mathfrak{a}$; i.e., the operad $\Lambda \mathfrak{a}$ characterized by the property that giving $\sigma C$ the structure of a coalgebra over $\mathfrak{a}$ is the same as giving $C$ the structure of a coalgebra over $\Lambda \mathfrak{a}$. Explicitly

$$
(\Lambda \mathfrak{a})(n)=\sigma^{1-n} \mathfrak{a}(n) \otimes \operatorname{sgn}_{n},
$$

where $\operatorname{sgn}_{n}$ is the sign representation of $\Sigma_{n}$ (see [12]).

Definition 4.4. The symmetric join operad $\{\mathfrak{j}(n)\}_{n \geqslant 0}$ is the operad $\mathfrak{j}=\Lambda \mathfrak{a}$ in the category of chain complexes. Explicitly

$$
\mathfrak{j}(n)=\sigma^{-n} C_{*}^{\mathcal{O}}\left(\mathcal{O} \Sigma_{n}, \partial \mathcal{O} \Sigma_{n}\right) \otimes \operatorname{sgn}_{n} .
$$

Theorem 4.5. The symmetric join operad $\{\mathfrak{j}(n)\}_{n \geqslant 0}$ is a unital $E_{\infty}$-operad of chain complexes. For any simplicial set $Y$ the chain complex $C_{*}(Y)$ is naturally a j-coalgebra (hence $C^{*}(Y)$ is a $\mathfrak{j}$-algebra).

Proof. Each $\mathfrak{j}(n)_{d}$ is clearly a free $k\left[\Sigma_{n}\right]$-module. Since $C_{d}^{\mathcal{O}}\left(\mathcal{O} \Sigma_{n}, \partial \mathcal{O} \Sigma_{n}\right)=0$ for $d<n$, each chain complex $\mathfrak{j}(n)$ is concentrated in non-negative degrees. Because $\left(\mathcal{O} \Sigma_{n}, \partial \mathcal{O} \Sigma_{n}\right)=\left(\mathcal{O}_{n}, \partial \mathcal{O}_{n}\right) \Sigma$, Proposition 4.1 provides for $n \geqslant 1$ a quasi-isomorphism

$$
C_{*}^{\mathcal{O}}\left(\mathcal{O} \Sigma_{n}, \partial \mathcal{O} \Sigma_{n}\right) \rightarrow C_{*}^{\mathcal{O}}\left(\mathcal{O}_{n}, \partial \mathcal{O}_{n}\right)=\sigma C_{*}\left(\Delta_{n-1}, \partial \Delta_{n-1}\right),
$$

where the last complex has one-dimensional homology group concentrated in degree $n$. Moreover $\mathfrak{j}(0)=k$. The other statements follow from the properties of the previously constructed operad $\mathfrak{a}$.

Let us make a few remarks.

- Since a surjective morphism in $\mathcal{O} \Sigma(\overline{n+1}, \bar{n})$ must send two elements of $\overline{n+1}$ to the same value and be injective otherwise, one can observe that $\mathfrak{j}(n)_{0}=k\left[\Sigma_{n}\right]$ splits as a direct sum

$$
\mathfrak{j}(n)_{0}=k\left[\operatorname{id}_{n}-\operatorname{sgn}(\pi) \pi\right]_{\pi \in \Sigma_{n}} \oplus k\left[\mathrm{id}_{n}\right]
$$

where additionally the first summand is precisely the image of the differential $d: \mathfrak{j}(n)_{1} \rightarrow \mathfrak{j}(n)_{0}$. It follows that the sign map

$$
\operatorname{sgn}: \mathfrak{j}(n)_{0} \rightarrow k
$$

is an augmentation of $\mathfrak{j}(n)$ and it defines a quasi-isomorphism $\mathfrak{j} \rightarrow \mathfrak{C o m}$ to the commutative operad. 
- The degree zero maps

$$
\mathfrak{j}(n)_{0} \otimes \mathfrak{j}\left(k_{1}\right)_{0} \otimes \cdots \otimes \mathfrak{j}\left(k_{n}\right)_{0} \rightarrow \mathfrak{j}\left(k_{1}+\cdots+k_{n}\right)_{0}
$$

agree, up to sign, with the maps

$$
k\left[\Sigma_{n}\right] \otimes k\left[\Sigma_{k_{1}}\right] \otimes \cdots \otimes k\left[\Sigma_{k_{n}}\right] \rightarrow k\left[\Sigma_{k_{1}+\cdots+k_{n}}\right]
$$

induced by the canonical permutation operad $\left\{\Sigma_{n}\right\}_{n \geqslant 0}$ in the category of sets [5, Sec. 0.10].

- The general framework of Berger [4], adapted to chain complexes in [17, Sec. 5], enables us to construct a filtration of the symmetric join operad by sub- $E_{n^{-}}$ operads.

For $f \in \mathcal{O} \Sigma(\overline{k+d}, \bar{k})$ let $s f \in \mathcal{O} \Sigma(\overline{k+d+1}, \bar{k})$ be the morphism defined by requiring that $(s f)(1)=1,(s f) \circ \delta_{1}=f$, and that 1 is the smallest element in the total ordering of $(s f)^{-1}(1)$. A direct verification shows that $s$ determines a chain homotopy $s: \mathfrak{j}(k)_{d} \rightarrow \mathfrak{j}(k)_{d+1}$, which is a chain contraction of $\mathfrak{j}(k)$ onto a chain subcomplex isomorphic to $\mathfrak{j}(k-1)$. Now define a cell structure on $\mathfrak{j}$ by lifting the basis of the cell structure on the sequence operad (defined using "complexity" in [17, Def. 5.5]) via the forgetful functor $\mathcal{O} \Sigma \rightarrow$ Set. Using the chain homotopy $s$, one shows that the cells are contractible as in [17, Prop. 5.6]. The remaining arguments in [17, Sect. 5], which show how to assemble the cells into a filtration by $E_{n}$-operads, go through without change, with the proviso that a "map" should be understood as "the underlying map of a morphism in $\mathcal{O} \Sigma . "$

\section{Proofs.}

This section contains all the postponed proofs. They are expressed using the interpretation of morphisms in $\mathcal{O} \Sigma$ as set maps $f$ with ordering on each fibre and rely on the notation and properties $(\mathcal{P} 1)-(\mathcal{P} 4)$ of Section 2.2. We fix one more convention. Given a set $\overline{k_{1}+\cdots+k_{n}}$ the pair $(i, j)$ denotes the $j$-th position in the $i$-th summand; i.e., the element $k_{1}+\cdots+k_{i-1}+j$.

\subsection{Proof of Theorem 3.3.}

It is easy to check that the maps (11) are equivariant with respect to the actions of $\Sigma_{n}$ and $\left(\Sigma_{k_{1}} \times \cdots \times \Sigma_{k_{n}}\right)$. We proceed to verify the operadic associativity axiom. Consider the family $\mathcal{O} \Sigma_{k_{i, j}}$ of $\mathcal{O} \Sigma$-sets, where $i=1, \ldots, n$ and $j=1, \ldots, k_{i}$ for some $k_{i}$. We need to show the commutativity of the outermost rectangle in the diagram

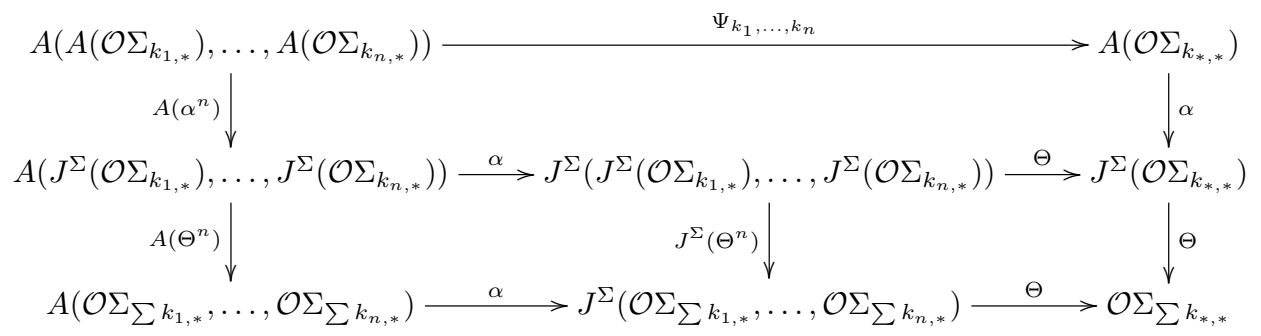


In order to streamline notation it will be convenient to show the commutativity of the three rectangles separately.

First consider the top rectangle. Note that the degree $k$ component of $A\left(X_{*}\right)$ can also be written as

$$
A\left(X_{*}\right)(k)=\coprod_{f \in \mathcal{O} \Sigma(\bar{k}, \bar{n})} \prod_{i=1, \ldots, n} X_{i}(k) .
$$

Suppose we start in the summand of the upper-left corner indexed by the collection $\left(f ; g_{1}, \ldots, g_{n}\right)$. Both ways of going around the diagram send this summand to the summand indexed by

$$
h=f\left\langle g_{1} \circ i_{f^{-1}(1)}, \ldots, g_{n} \circ i_{f^{-1}(n)}\right\rangle .
$$

The map $\Theta \circ \alpha \circ A\left(\alpha^{n}\right)$ acts on each individual factor $\mathcal{O} \Sigma_{k_{i, j}}$ of that summand by the $\mathcal{O} \Sigma$-morphism

$$
\xi_{1}=i_{g_{i}^{-1}(j)} \circ\left(i_{f^{-1}(i)}\right)^{g_{i}^{-1}(j)} \circ \mathrm{id} .
$$

On the other hand, the map $\alpha \circ \Psi_{k_{1}, \ldots, k_{n}}$ acts on $\mathcal{O} \Sigma_{k_{i, j}}$ via the morphism

$$
\xi_{2}=\mathrm{id} \circ i_{h^{-1}(i, j)} .
$$

We have the equality

$$
\xi_{1}=i_{g_{i}^{-1}(j)} \circ\left(i_{f^{-1}(i)}\right)^{g_{i}^{-1}(j)} \circ \mathrm{id}=i_{f^{-1}(i) \cap g_{i}^{-1}(j)}=i_{h^{-1}(i, j)}=\xi_{2},
$$

where the first transition follows from $(\mathcal{P} 4)$ and the second from the equality of sets $h^{-1}(i, j)=f^{-1}(i) \cap g_{i}^{-1}(j)$. This proves that the top rectangle commutes.

The bottom-left corner commutes because $\alpha$ is a natural transformation.

Consider now the bottom-right square (note that all maps in that square are isomorphisms). The degree $k$ component of $J^{\Sigma}\left(J^{\Sigma}\left(\mathcal{O} \Sigma_{k_{1, *}}\right), \ldots, J^{\Sigma}\left(\mathcal{O} \Sigma_{k_{n, *}}\right)\right)$ is

$$
\coprod_{f \in \operatorname{Set}(\bar{k}, \bar{n})} \prod_{i=1, \ldots, n} \coprod_{g_{i} \in \operatorname{Set}\left(\overline{\left.\left|f^{-1}(i)\right|, \overline{k_{i}}\right)}\right.} \prod_{j=1, \ldots, k_{i}} \mathcal{O} \Sigma\left(\overline{\left|g_{i}^{-1}(j)\right|}, \overline{k_{i, j}}\right) .
$$

Consider an element of this set given by a collection

$$
\begin{gathered}
f \in \operatorname{Set}(\bar{k}, \bar{n}), \quad\left\{g_{i} \in \operatorname{Set}\left(\overline{\left|f^{-1}(i)\right|}, \overline{k_{i}}\right)\right\}_{i=1, \ldots, n}, \\
\left\{h_{i, j} \in \mathcal{O} \Sigma\left(\overline{\left|g_{i}^{-1}(j)\right|}, \overline{k_{i, j}}\right)\right\}_{(i, j) \in \overline{k_{1}+\cdots+k_{n}}} .
\end{gathered}
$$

The two ways of going to $\mathcal{O} \Sigma_{\sum k_{*, *}}(k)$ send this family, respectively, to the morphisms

$$
\left(f\left\langle g_{1}, \ldots, g_{n}\right\rangle\right)\left\langle h_{1,1}, \ldots, h_{1, k_{1}}, \ldots, h_{n, 1}, \ldots, h_{n, k_{n}}\right\rangle
$$

and

$$
f\left\langle g_{1}\left\langle h_{1,1}, \ldots, h_{1, k_{1}}\right\rangle, \ldots, g_{n}\left\langle h_{n, 1}, \ldots, h_{n, k_{n}}\right\rangle\right\rangle,
$$

which are easily seen to be equal as elements of $\mathcal{O} \Sigma\left(\bar{k}, \overline{\sum k_{*, *}}\right)$.

\subsection{Proof of Proposition 4.3.}

Each of the maps used in the definition of $\mathfrak{a}_{n}^{X}$ is $\Sigma_{n}$-equivariant, hence so is the composition. For $C$ to be a coalgebra over $\mathfrak{a}$, we need the commutativity of the 
diagram

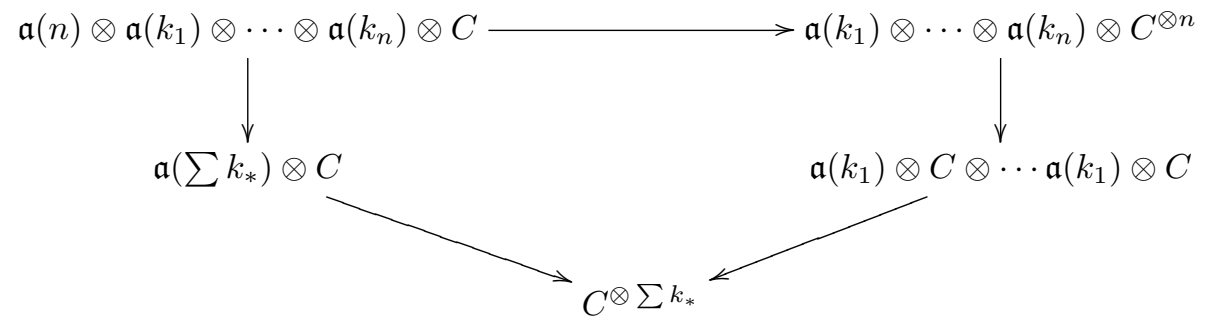

For this we first generalize the combinatorial "coaction" maps (16). For any sequence $X_{1}, \ldots, X_{n}$ of $\mathcal{O} \Sigma$-sets and integers $k_{1}, \ldots, k_{n}$ we have a map

$$
\mathcal{O} \Sigma_{k_{1}} \times \cdots \times \mathcal{O} \Sigma_{k_{n}} \times J^{\Sigma}\left(X_{1}, \ldots, X_{n}\right) \rightarrow J^{\Sigma}\left(\mathcal{O} \Sigma_{k_{1}} \times X_{1}, \ldots, \mathcal{O} \Sigma_{k_{n}} \times X_{n}\right) .
$$

On components of degree $k$

$$
\begin{aligned}
\mathcal{O} \Sigma\left(\bar{k}, \overline{k_{1}}\right) \times \cdots & \times \mathcal{O} \Sigma\left(\bar{k}, \overline{k_{n}}\right) \times \coprod_{f \in \operatorname{Set}(\bar{k}, \bar{n})} \prod_{i=1}^{n} X_{i}\left(\left|f^{-1}(i)\right|\right) \\
& \rightarrow \coprod_{f \in \operatorname{Set}(\bar{k}, \bar{n})} \prod_{i=1}^{n} \mathcal{O} \Sigma\left(\overline{\left|f^{-1}(i)\right|, \overline{k_{i}}}\right) \times X_{i}\left(\left|f^{-1}(i)\right|\right),
\end{aligned}
$$

it is given by the formula

$$
\left(g_{1}, \ldots, g_{n} ; x_{1}, \ldots, x_{n}\right) \rightarrow\left(\left(g_{1} \circ i_{f^{-1}(1)}, x_{1}\right), \ldots,\left(g_{n} \circ i_{f^{-1}(n)}, x_{n}\right)\right) .
$$

By post-composing the map of (27) with the "coactions" $\psi_{k_{i}}^{X_{i}}$ of (16), we obtain maps

$$
\widetilde{\psi}: \mathcal{O} \Sigma_{k_{1}} \times \cdots \times \mathcal{O} \Sigma_{k_{n}} \times J^{\Sigma}\left(X_{1}, \ldots, X_{n}\right) \rightarrow J^{\Sigma}\left(J^{\Sigma}\left(X_{1}^{k_{1}}\right), \ldots, J^{\Sigma}\left(X_{n}^{k_{n}}\right)\right),
$$

which satisfy the following compatibility.

Lemma 5.1. For any $\mathcal{O} \Sigma$-set $X$ and integers $n, k_{1}, \ldots, k_{n}$ the following diagram of $\mathcal{O} \Sigma$-sets commutes.

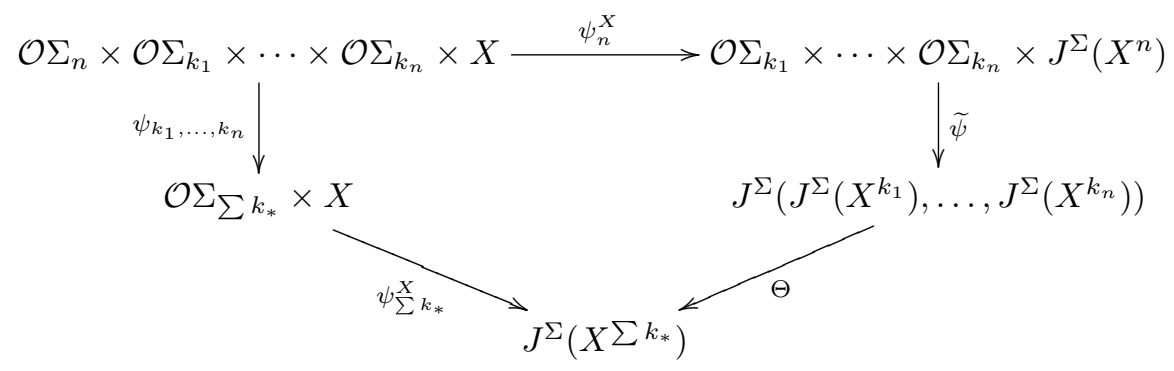

Proof. The proof resembles that of Section 5.1 and uses (12), (13), (8), the map $\Theta$ from 3.2, and (28). Consider a tuple $\left(f ; g_{1}, \ldots, g_{n} ; x\right)$ in $\mathcal{O} \Sigma_{n} \times \mathcal{O} \Sigma_{k_{1}} \times \cdots \times \mathcal{O} \Sigma_{k_{n}} \times$ $X$. Each of the two ways around the diagram sends it to the summand of the join 
indexed by the map

$$
h=f\left\langle g_{1} \circ i_{f^{-1}(1)}, \ldots, g_{n} \circ i_{f^{-1}(n)}\right\rangle .
$$

The result in $J^{\Sigma}\left(X^{\sum k_{*}}\right)$ is a tuple indexed by pairs $(i, j), 1 \leqslant i \leqslant n, 1 \leqslant j \leqslant k_{i}$. The left path in the diagram produces a tuple whose $(i, j)$-th entry is

$$
x \circ i_{h^{-1}(i, j)},
$$

while for the other path it is

$$
\begin{aligned}
x \circ i_{f^{-1}(i)} \circ i_{\left(g_{i} \circ i_{f^{-1}(i)}\right)^{-1}(j)} & =x \circ i_{f^{-1}(i)} \circ i_{\left(i_{f^{-1}(i)}\right)^{-1}\left(g_{i}^{-1}(j)\right)} \\
& =x \circ i_{g_{i}^{-1}(j)} \circ\left(i_{f^{-1}(i)}\right)^{g_{i}^{-1}(j)},
\end{aligned}
$$

where the last equality follows from $(\mathcal{P} 1)$. We conclude that the two maps are equal as in Section 5.1.

To complete the proof of Proposition 4.3, note that Lemma 5.1 gives, after application of the chains functor $C_{*}^{\mathcal{O}}(\cdot)$, the following commutative diagram for every $\mathcal{O}$-set $X$. In the diagram $s$ is the map of Proposition 4.2.

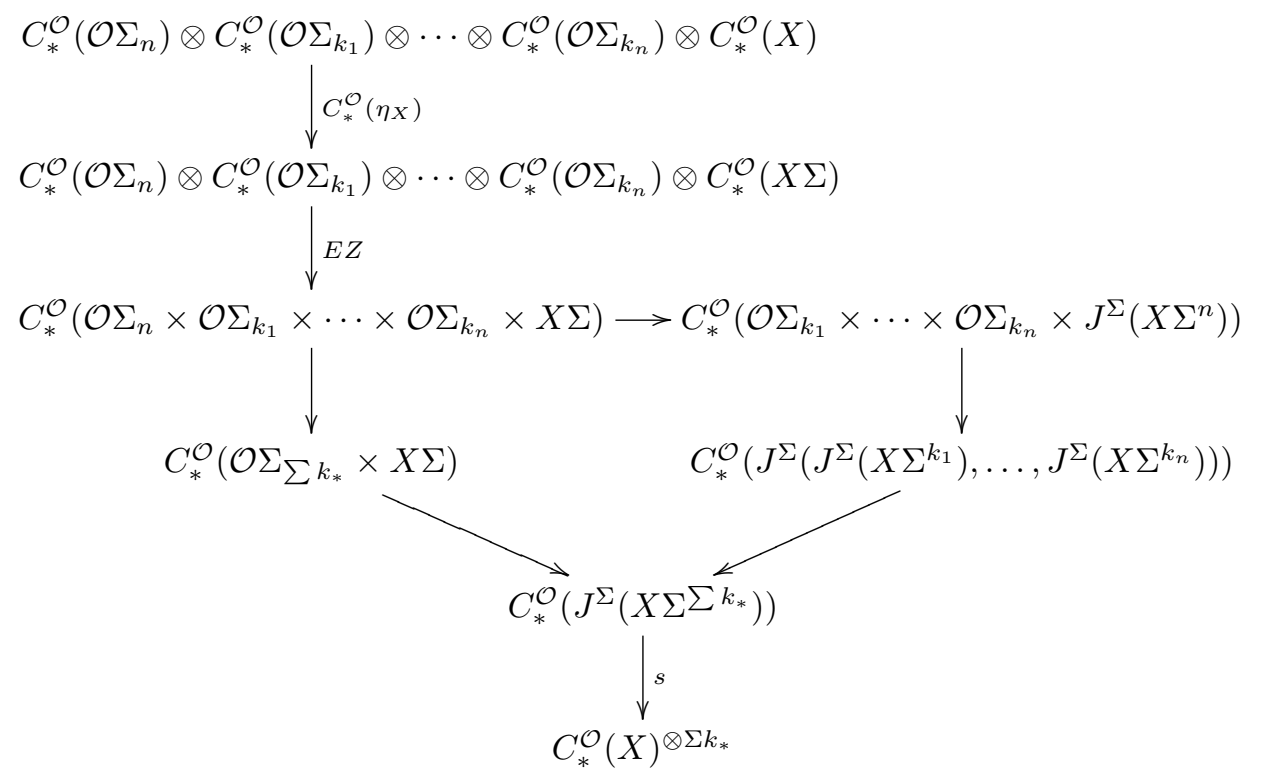

It follows that the same diagram commutes for the relative objects $(\mathcal{O} \Sigma, \partial \mathcal{O} \Sigma)$, $(X, X(0))$, and $(X \Sigma, X \Sigma(0))$, and the two ways of traversing that diagram correspond to the two ways around (26) for $C=C_{*}^{\mathcal{O}}(X, X(0))$.

That ends the proof.

\section{Acknowledgments}

The work of the first author was carried out while at the Centre for Discrete Mathematics and its Applications (DIMAP), Mathematics Institute, University of Warwick, Coventry, CV4 7AL, United Kingdom. The support of DIMAP via the EPSRC award EP/D063191/1 is gratefully acknowledged. 
We are grateful to the referee for a number of suggestions, especially concerning the Day convolution.

\section{References}

[1] M.A. Batanin and C. Berger, The lattice path operad and Hochschild cochains, Contemp. Math. 504 (2009), 23-52.

[2] M.A. Batanin, C. Berger, and M. Markl, Operads of natural operations I: Lattice paths, braces and Hochschild cochains, SMF Congres et Seminaires 26 (2011), 1-33.

[3] M.A. Batanin and M. Markl, Crossed interval groups and operations on the Hochschild cohomology, preprint arXiv/0803.2249.

[4] C. Berger, Combinatorial models for real configuration spaces and $E_{n}$-operads, Contemp. Math. 202 (1997), 37-52.

[5] C. Berger and B. Fresse, Combinatorial operad actions on cochains, Math. Proc. Cambridge Philos. Soc. 137 (2004), 135-174.

[6] B.J. Day, On closed categories of functors, Lecture Notes in Mathematics 137 (1970), 1-38.

[7] B.J. Day and R. Street, Lax monoids, pseudo-operads, and convolution, in Diagrammatic Morphisms and Applications, Contemporary Mathematics vol. 318, AMS, April 2003, 75-96.

[8] W.G. Dwyer, M.J. Hopkins, and D.M. Kan, The homotopy theory of cyclic sets, Trans. AMS 291, no. 1 (Sep. 1985), 281-289.

[9] W.G. Dwyer and J. Spalinski, Homotopy theories and model categories, in I.M. James (ed.), Handbook of Algebraic Topology, Elsevier, Amsterdam 1995.

[10] P.J. Ehlers and T. Porter, Joins for (augmented) simplicial sets, Journal of Pure and Applied Algebra 145, issue 1 (January 2000), 37-44.

[11] Z. Fiedorowicz and J.-L. Loday, Crossed Simplicial Groups and their Associated Homology, Trans. A.M.S. 326, no. 1 (July 1991), 57-87.

[12] J.D.S. Jones, Lectures on operads, Contemporary Mathematics 315, 89-130.

[13] M. Mandell, $E_{\infty}$-algebras and p-adic homotopy theory, Topology 40 (2001), $43-94$.

[14] M. Mandell, Cochains and homotopy type, Publ. Math. Inst. Hautes Etudes Sci. 103 (2006), 213-246.

[15] M. Markl, S. Shnider, and J. Stasheff, Operads in Algebra, Topology and Physics, Mathematical Surveys and Monographs, AMS, Providence, RI, 2002.

[16] J.P. May, Simplicial objects in algebraic topology, Van Nostrand, New York, 1967.

[17] J. McClure and J.H. Smith, Multivariable cochain operations and little n-cubes, J. Amer. Math. Soc. 16 (2003), 681-704. 
[18] T. Pirashvili, On the PROP corresponding to bialgebras, Cahiers de topologie et geometrie differentielle categoriques 43 (2002), 221-239.

[19] N.E. Steenrod, Products of cocycles and extensions of mappings, Ann. of Math. (2) 48 (1947), 290-320.

Michał Adamaszek aszek@mimuw.edu.pl

Mathematics Institute, Universität Bremen, Bibliothekstr. 1, 28359 Bremen, Germany

John D.S. Jones J.D.S. Jones@warwick.ac.uk

Mathematics Institute, University of Warwick, Coventry, CV4 7AL, UK 